\title{
Talento y conflictos en el clima organizacional en escuelas públicas
}

\author{
Betty Quispe Yucra \\ betty24qy@gmail.com \\ ORCID.0000-0002-6757-176X \\ Universidad Privada César Vallejo \\ Lima - Perú
}

\section{RESUMEN}

El estudio fue de tipo básica, bajo el diseño no experimental, correlacional, porque se determinó la influencia entre las variables de estudio, apoyándose en el método hipotético deductivo, la población de estudio ha sido 635 docentes de escuelas públicas de Lima y una muestra de 240 docentes encuestados, determinado por el método no probabilístico censal por conveniencia, se utilizaron tres cuestionarios, una para cada variable de estudio, el análisis de los datos se realizó utilizando el algoritmo de regresión logística multinomial. Los resultados indican que la gestión del talento y conflictos tienen influencia significativa en el clima organizacional de las públicas, como lo demuestra la prueba del pseudo $\mathrm{R}$ cuadrado, la misma que expresa la dependencia porcentual de la gestión del talento y conflictos en el clima organizacional para lo cual se tiene al coeficiente de Nagelkerke, implicando que la variabilidad del clima organizacional depende el $55.1 \%$ de la gestión del talento y conflictos.

Palabras claves: gestión del talento; gestión; conflictos; clima organizacional. 


\title{
Talent and conflicts in the organizational climate in public schools
}

\begin{abstract}
The study was of a basic type, under the non-experimental, correlational design, because the influence between the study variables was determined, relying on the hypothetical deductive method, the study population has been 635 teachers from public schools in Lima and a sample of 240 teachers surveyed, determined by the non-probabilistic convenience census method, three questionnaires were used, one for each study variable, the data analysis was performed using the multinomial logistic regression algorithm. The results indicate that talent and conflict management have a significant influence on the organizational climate of public companies, as shown by the pseudo R-squared test, which expresses the percentage dependence of talent and conflict management on the organizational climate for which is the Nagelkerke coefficient, implying that the variability of the organizational climate depends $55.1 \%$ on the management of talent and conflicts.
\end{abstract}

Keywords: talent management; management; conflict, organizational climat.

Artículo recibido: 30 noviembre. 2021 Aceptado para publicación: 29 diciembre 2021

Correspondencia: betty24qy@gmail.com Conflictos de Interés: Ninguna que declarar 


\section{INTRODUCCIÓN}

En palabras de Bustamante et al., (2015) que señalan a (Bernal, Pedraza y Sánchez, 2015) sustenta que el clima organizacional tiene un impacto en la calidad de los servicios y ésta se convierte en el indicador de un clima organizacional óptimo. En el estudio se han considerado algunas características del clima organizacional como la motivación que muestran los colaboradores, la calidad de su enseñanza y la comunicativa asertiva que promueve el desarrollo organizacional. En la gestión de las escuelas públicas un aspecto a considerar es la cultura organizacional, toda organización se conduce por los lineamientos como valores, misión y visión, otro aspecto importante es la formación del servidor en el desarrollo de tareas las cuales pasan por una evaluación de desempeño. Cabe mencionar que desarrollar tareas en una organización desgasta la calidad de vida, la satisfacción y el trabajo colaborativo de los que prestan el servicio educativo que terminan generando conflictos al interior de la organización

La gestión de las escuelas públicas básicas peruanas se rige por normas emanadas por el Ministerio de Educación, con una línea de organización jerárquica que funciona de acuerdo a la normativa, se mencionan tres documentos Ley de la Reforma Magisterial, Marco del Buen Desempeño Directivo, Marco de Buen Desempeño del Docente, en ellos el capital humano es fundamental para el logro de competencias, así mismo es necesario indicar el Currículo Nacional de la Educación Básica que está alineado al Proyecto Educativo Nacional y a los objetivos de la educación básica e inciden en la satisfacción de los usuarios del servicio educativo.

El objetivo general de la investigación señalar relación entre el talento y conflictos en el clima laboral en las escuelas públicas, el objetivo específicos a)Señalar la relación del talento y conflictos en la motivación de las escuelas, b)Señalar la relación del talento y conflictos en el liderazgo de las escuelas y c)Señalar la relación del talento y conflictos en la comunicación de las escuelas.

La hipótesis general. La gestión del talento y conflictos tienen influencia significativa en el clima organizacional de las escuelas. Hipótesis específicas a)la gestión del talento y conflictos tiene influencia significativa en la motivación de las escuelas, b)La gestión del talento y conflictos tiene influencia significativa en el liderazgo de las escuelas y c)la gestión del talento y conflictos tiene influencia significativa en la comunicación de las escuelas. 
En opinión de Sarabia (2014) para puntualizar el clima organizacional es necesario considerar dos aspectos: los colaboradores y el ambiente como parte adicional y conjuntivas de un hecho. Haciendo un análisis del clima laboral menciona a Brunet (2004) quien señala que la conducta de la persona es vista desde tres puntos a) cualidades organizacionales, b) percepción de las cualidades organizacionales y c) percepción de las cualidades individuales sobre la organización. Para referirse a las dimensiones del clima organizacional Sarabia (2014) manifiesta que es multidimensional y nombra a Rivas (1992) quienes dimensionan en siete aspectos: a) ambiente físico de trabajo, b) comunicación e interacción c) incentivación, d) Identificación y pertenencia, e) autoridad y liderazgo, f) control y supervisión y g) motivación laboral.

En su investigación Bowie, (2013) manifiesta que un elemento fundamental para la solidez organizacional es una adecuada atmósfera moral, que descansa en normas y valores, como también el compromiso con la administración de las partes en interés, es decir considerar el objetivo de la organización y el papel de los estímulos, porque fortalecen o retraen la integridad organizacional, reconocen aspectos que suman en contra de la integridad moral.

Según Yazdi \& Yakhdani (2017) define al clima organizacional puntualizando dos aspectos: sistema cognitivo que trasluce la cultura organizacional (visión, misión, valores) y otro elemento emocional que emana de la política, del ejercicio y procedimientos organizacionales que están ligadas al liderazgo. El estudio hace mención también a Moran y Volkwayne citados por Khosravian et al. determinan cuatro enfoques a) Estructural, los colaboradores comparten características de configuración de la organización. b) Conceptual, los colaboradores responden a las situaciones versátiles de la organización. c)Interactivo, mediante variadas situaciones que se presentan llegan a acuerdos institucionales. d)Cultural, promueve una interacción entre colaboradores y como resultado en la organización comparten un estado de ánimo.

En su estudio Madero \& Olivas (2016) haciendo la exploración de las particularidades del comportamiento organizacional en colaboradores que inician su vida profesional refiriéndose a la motivación menciona a Arrieta y Navarro (2008) como la fuerza psicológica que actúa desde lo profundo de cada ser y le indica el rumbo del comportamiento, considerando los niveles de esfuerzo y persistencia de la conducta sin estar sujeta a presiones y limitaciones contextuales poniendo intensidad en las 
interacciones entre el ser (intereses, necesidades y valores) y el medio (tipo de tareas, condiciones laborales).

Liderazgo en opinión de (Sills, 1968) señala cuando un ser ejerce influencia sobre otro, o casi siempre, que uno o algunos escasos personajes condicen a un grupo mayor. Un aspecto común del liderazgo es que algunas personas influyen en los demás algunas veces o casi siempre y muchas veces algunos pocos influyen en muchos y muchas veces. El ser guiado por un líder implica compartir una dirección común, por tanto, muy generalmente corresponde al grupo que son parte del liderazgo que tienen un objetivo general similares o comunes.

Considerando las indagaciones de Sardón \& Sardón (2018) las disputas se presentan entre partes donde una se beneficia y la otra se perjudica, así mismo señala la relación directa con el medio organizacional y cuando este es desfavorable y deja de satisfacer a los copartícipes, otro indicador de conflictos al interior de la organización son las posturas antagónicas entre los copartícipes.

El conflicto se da entre dos partes donde una percibe y la otra se afecta, así mismo indica que hay una relación directa con la atmósfera organizacional y cuando este es adverso y no colma las necesidades de los colaboradores. Menciona también que un indicador de conflictos al interior de las organizaciones son las posiciones contrarias.

Según Morrill (2000) Existen dos formas de usar el conflicto en las organizaciones: uno para reprimir donde hay una situación manifiesta y disfuncional convirtiéndose en una amenaza para la productividad, el confort de los colaboradores y de los líderes; dos las desavenencias son usadas de manera fructífera.

En opinión de Moran (2020) en su estudio considera el estrés laboral para iniciar su investigación y los enfrentamientos son muestras del agotamiento físico y mental de los docentes, lo que repercute en la desatención de su labor pedagógica y gestándose un medio propicio para situaciones conflictivas con sus pupilos, pares, padres de familia y líderes.

Considerando el estudio de Piva \& Padua (2015) quienes aluden a Franca (1997) describe que la calidad de vida laboral se define como el conjunto de acciones de una organización que implica transportar a las actividades cotidianas el perfeccionamiento en cambios de gestión y tecnologías en la zona laboral. La calidad de vida laboral se edifica desde la 
mirada conjunta de colaboradores y líderes en la organización, es decir considerando una óptica biopsicosocial.

En un estudio respecto a la gestión y dirección del talento, Cardona et al. (2018) indica que un descuido de las organizaciones es dejar de observar a los colaboradores como pieza elemental de los centros de formación educativa, es importante saber que el educador emane los valores organizacionales, la superioridad a través del servicio que prestan a los usuarios; por tanto, es notable que el clima organizacional deje de ser ignorado por las instituciones, a su vez de recibir los recursos necesarios y el acompañamiento imprescindible que garantice el desempeño íntegro del docente.

En opinión de Serra (2021) para referirse a las extensión o dimensiones del manejo del talento realiza una división en dos partes, definir qué es el talento y cuál es la metodología para gestionar su desarrollo en función a estos dos pilares las organizaciones toman decisiones que fortalecen su administración y crecimiento. El autor sostiene que la organización crea el talento en el que cree, en consecuencia, la organización genera el desarrollo del talento y esta afirmación la sostiene indicando que en pleno siglo XXI las instituciones tecnológicamente más desarrolladas no necesariamente son las más exitosas, por tanto, en este siglo lo que marca la diferencia son los colaboradores, es decir el talento gestionado y desarrollado por las organizaciones.

\section{ESTRATEGIAS METODOLÓGICAS O MATERIALES Y MÉTODOS}

Es una investigación de tipo descriptivo, diseño no experimental de corte transversal, el método de trabajo es hipotético deductivo la población a sido 635 docentes y una muestra de 240 docentes encuestados, determinado por el método no probabilístico censal por conveniencia, para la recopilación de datos se utilizaron tres cuestionarios. El análisis de los datos se realizó utilizando el algoritmo de regresión logística multinomial

\section{RESULTADOS Y DISCUSIÓN}

Análisis descriptivo por variable y dimensiones

Tabla 1 Frecuencias y porcentajes de los niveles de la variable gestión de talento

\begin{tabular}{ccc}
\hline Niveles & Frecuencia & Porcentaje \\
\hline Bajo & 4 & $1.7 \%$ \\
Medio & 25 & $10.4 \%$ \\
Alto & 211 & $87.9 \%$ \\
Total & 240 & $100 \%$ \\
\hline
\end{tabular}

Fuente: Elaboración propia, 2021. 
Tabla 2 Frecuencias y porcentajes de los niveles de la variable gestión de talento por dimensiones

\begin{tabular}{lccccccccc}
\hline \multicolumn{1}{c}{ Dimensiones } & \multicolumn{2}{c}{ Bajo } & \multicolumn{2}{c}{ Medio } & \multicolumn{2}{c}{ Alto } & \multicolumn{2}{c}{ Total } \\
\hline & f & \% & f & $\%$ & f & $\%$ & f & $\%$ \\
\hline Cultura organizacional & 7 & 2.9 & 37 & 15.4 & 196 & 81.7 & 240 & 100 \\
Conocimiento específico & de & 6 & 2.5 & 35 & 14.6 & 199 & 82.9 & 240 & 100 \\
las tareas del puesto & & & & & & & & \\
Evaluación de desempeño & 10 & 4.2 & 164 & 68.3 & 66 & 27.5 & 240 & 100 \\
\hline
\end{tabular}

Fuente: Elaboración propia, 2021.

En cuanto los resultados descriptivos, los hallazgos evidenciaron que los docentes de las de las escuelas públicas en su mayoría, es decir $87.9 \%$ consideraron que la gestión de talento influye significativamente en el clima organizacional dando un alto nivel, este resultado es respaldad por el estudio de Cardona et al. (2018) cada colaborador posee sus peculiaridades en su desenvolvimiento, condicionado por el entorno laboral, la estructura y el ambiente que hay en la organización, estos elementos inciden directamente en la práctica laboral, permitiendo además que el docente tenga la oportunidad de mejorar y que vea respaldada su contribución al interior de la institución en un medio de confianza y respeto. Eckardt et al., (2017) centró su estudio en identificar niveles de creatividad y habilidades al interior de las corporaciones, que brinden oportunidades para la integración, así mismo buscó incrementar el entendimiento sobre el capital humano, valiéndose de dos corrientes de investigación que se centran directamente en el capital humano estratégico (SHC) y recurso humano estratégico HRM. Otra mirada del estudio es la administración del capital humano vinculadas con los resultados institucionales e individuales. McDonnell (2011) cuyo propósito es identificar dificultades críticas en las formas de dirigir las habilidades humanas, como resultado encontró que la gestión del talento viene a ser el reto más grande que enfrentarán las organizaciones en las siguientes décadas y que la demografía de la fuerza laboral y la escasez de habilidades hagan que la “guerra por el talento" sea más feroz y que la gestión eficaz del talento sea una necesidad competitiva.

Los resultados descriptivos de las dimensiones de la variable Gestión de talento son altas respecto a la variable Clima organizacional, si detallamos los resultados de cultura 
organizacional tiene una relación significativa que arroja un $81,7 \%$ entendiendo que cultura organizacional comprende el conocimiento de la misión y objetivos de la organización cultura organizacional, es decir conocer los documentos de gestión institucionales como el PEI donde se encuentran los aspectos propios de una cultura organizacional, que generan identidad con la organización y está relacionada con el reconocimiento y felicitación, así como las posibilidades de crecimiento profesional al interior de la organización que recibe un colaborador . Bowie, (2013) sostiene que la clave para la integridad organizacional es un buen clima moral, basado en normas y valores, así mismo un compromiso con la gestión de las partes interesadas, un compromiso de ver el propósito de la organización.

Por otro lado, la dimensión conocimiento específico de las tareas del puesto arrojó un resultado de $82,9 \%$ respecto al clima organizacional esto nos indica que todo docente que ingresa en la carrera magisterial está acreditado y cumple con una serie de requisitos para acceder al puesto y están dirigidas por el órgano rector como es el MINEDU según lo expuesto para sustentar el resultado nos apoyamos en la opinión de Chiavenato (2009), un puesto de trabajo es un espacio de labores para un trabajador que desarrolla una determinada tarea o función laboral en el puesto y se espera que el empleado desempeñe el rol con eficiencia y eficacia y que sea apreciada a través de su productividad para la organización sintiendo ésta que los objetivos organizacionales se desarrollan.

Así mismo la dimensión evaluación de desempeño nos arrojó un resultado de 68,3\% considerado como bueno y nos indica la relación positiva con el clima organizacional. El conocimiento específico de las tareas y la evaluación de desempeño son elementos correlacionados con el clima organizacional siendo así un indicadores de desarrollo organizacional, esta afirmación podemos corroborar con el estudio realizado por Jee Kim \& Park, (2018) quien determinan que las relaciones entre la administración con apoyo al talento humano dentro de una cultura de organización indica una asociación positiva entre la complacencia de los colaboradores y el empresariado mostrando resultados mediados por la cultura organizacional. Así mismo Chiavenato (2009) la administración del talento humano se transformó en un elemento imprescindible que garantiza el prestigio de las instituciones. Los colaboradores con talentos muestran algunas características como: a) Conocimiento, constituye uno de los recursos fundamentales del hombre de esta era como es el saber, empoderado que se sostiene en la educación y la filosofía de aprender a 
aprender. b) Habilidad, la pericia con la cual aplica su saber para solucionar problemas, inventar, modernizar situaciones que se traduce en el saber hacer. c) Juicio, la capacidad de examinar el contexto, la situación donde se desempeñan usando su espíritu crítico, ponderación, precisando prioridades, identificando datos e información. d)Actitud, proceder decidido para conseguir metas, tomar riesgos procediendo como representante de cambios, dando valor, buscando la excelencia y éxito. Toulson \& Smith (1994) "Existe evidencia que sugiere que el clima organizacional puede influye tanto en el desempeño laboral como en la satisfacción de los empleados" si hay colaboradores contentos, se pueden promover atmósferas adecuadas al interior de la organización. En este estudio se refleja la relación inversa de clima organizacional con la dimensión desempeño laboral una dimensión de la variable gestión de talento. Robbins y Judge (2013), mencionados en su estudio por (López Martínez \& Ibarra Cisneros, 2019) detallan que las personas.

Tabla 3 Frecuencia y porcentaje de los niveles de la variable conflictos

\begin{tabular}{ccc}
\hline Niveles & Frecuencia & Porcentaje \\
\hline Bajo & 0 & $33.8 \%$ \\
Medio & 81 & $66.2 \%$ \\
Alto & 240 & $100 \%$ \\
Total & 240 & \\
\hline
\end{tabular}

Fuente: Elaboración propia, 2021.

Tabla 4 Frecuencias y porcentaje de los niveles de la variable conflictos por dimensiones

\begin{tabular}{lcccccccc}
\hline \multicolumn{1}{c}{ Dimensiones } & \multicolumn{2}{c}{ Bajo } & \multicolumn{2}{c}{ Medio } & \multicolumn{2}{c}{ Alto } & \multicolumn{2}{c}{ Total } \\
\hline & f & \% & f & \% & f & \% & f & $\%$ \\
\hline Calidad de vida laboral & 2 & 0.8 & 71 & 29.6 & 167 & 69.6 & 240 & 100 \\
Satisfacción del docente & 8 & 3.3 & 201 & 83.8 & 31 & 12.9 & 240 & 100 \\
Trabajo en equipo & 2 & 0.8 & 92 & 38.6 & 146 & 60.8 & 240 & 100 \\
\hline
\end{tabular}

Fuente: Elaboración propia, 2021.

En cuanto los resultados descriptivos, de la variable conflictos los hallazgos evidenciaron que los docentes de las escuelas públicas en su mayoría, es decir $66.2 \%$ considerado como alta, evidenciando una relación significativa con el clima organizacional, este resultado sustentamos con la opinión de (Sardón Ari \& Sardón Ari, 2018) al interior de las escuelas los conflictos se producen como resultado de las divergencias entre individuos que sienten 
los acontecimientos de forma diferente en función a sus valores y paradigmas propios de cada uno en clara contraposición entre sus pares y alterar la atmósfera organizacional Chiavenato (2007) la forma de gestionar el conflicto determina "los resultados constructivos o destructivos" e interfieren directamente en el clima organizacional.

Respecto a los resultados de la dimensión Calidad de vida laboral arrojó un 69,6\% lo que indica la relación significativa de esta dimensión con el clima organizacional y apoyamos este resultado con el estudio Chiavenato (2007) una corporación es competitiva cuando los colaboradores se encuentran laborando en el mejor lugar, con las mejores circunstancias de salubridad, higiene y condiciones de seguridad psicológica y social con un orden fabuloso, en efecto motivados y con la más alta productividad. La gestión de la sumatoria de la calidad organizacional es consecuencia de la optimización de la excelencia humana y está relacionado de lo bien que se siente un colaborador en la organización.

Los resultados de la dimensión satisfacción del docente de la variable conflicto arrojó como medio $83,8 \%$ lo que es considerada como muy bueno mostrándonos la relación significativa de la satisfacción docente con el clima organizacional resultado que apoyamos en las opiniones de Maxwell (2014) "La satisfacción laboral es la sensación de logro y éxito de un trabajador en el trabajo" cuando el colaborador se siente a gusto disfruta de la labor que realiza, lo hace bien y recibe una remuneración, por tanto, está relacionado de forma lineal con el rendimiento. Todo trabajador necesita auto valorarse, sentirse necesario, identificarse con la organización para reforzar la satisfacción laboral. Por otro lado, el beneficio para la organización es "los altos niveles de satisfacción laboral de sus trabajadores sugieren fuertemente una fuerza laboral motivada y comprometida con un desempeño de alta calidad".

Los resultados de la dimensión Trabajo en equipo de la variable conflictos nos arrojó un valor alto $60,8 \%$ considerada como muy alta, lo que nos manifiesta la relación significativa del trabajo en equipo con la variable clima organizacional, este resultado se apoya en los estudios realizado por (Herbert Ferrara, 2013) "Los equipos de personas que trabajan juntas hacia un objetivo proporcionan mejoras más permanentes en los procesos y operaciones que las personas que trabajan solas". Las personas que conforman grupos de trabajo "deben sentirse más cómodos" poniendo en la mesa de trabajo los problemas y obtener soluciones de sus colegas colaboradores en sustento de (Padhi, 2012), nombrado 
por Herbert, por otro lado, los directores plantean las formas o estrategias para impulsar el trabajo colaborativo e incrementar la producción que es deseada por la organización.

Tabla 5 Frecuencias y porcentajes de los niveles de la variable clima organizacional

\begin{tabular}{ccc}
\hline Niveles & Frecuencia & Porcentaje \\
\hline Bajo & 7 & $2.9 \%$ \\
Medio & 37 & $15.4 \%$ \\
Alto & 196 & $81.7 \%$ \\
Total & 240 & $100 \%$ \\
\hline
\end{tabular}

Fuente: Elaboración propia, 2021.

Tabla 6 Frecuencias y porcentajes de los niveles de la variable clima organizacional por dimensiones

\begin{tabular}{|c|c|c|c|c|c|c|c|c|c|}
\hline \multirow[t]{2}{*}{ Dimensiones } & & \multicolumn{2}{|c|}{ Bajo } & \multicolumn{2}{|c|}{ Medio } & \multicolumn{2}{|c|}{ Alto } & \multicolumn{2}{|c|}{ Total } \\
\hline & & f & $\%$ & $\mathbf{f}$ & $\%$ & $\mathbf{f}$ & $\%$ & $\mathbf{f}$ & $\%$ \\
\hline Motivación & & 8 & 3.3 & 160 & 66.7 & 72 & 30.0 & 240 & 100 \\
\hline Trabajo & & & & & & & & & \\
\hline Liderazgo & & 0 & 0.0 & 50 & 20.8 & 190 & 79.2 & 240 & 100 \\
\hline Comunicación & & 0 & 0.0 & 64 & 26.7 & 176 & 73.3 & 240 & 100 \\
\hline
\end{tabular}

Fuente: Elaboración propia, 2021.

El análisis descriptivo de la variable clima organizacional nos arrojó el siguiente resultado $81.7 \%$ de docentes de las escuelas públicas indican que el clima organizacional tiene una relación significativa con las variables gestión de talento y conflictos, estos resultados son sustentados con los estudios de Acosta \& Venegas, (2010) refiriéndose al enfoque cultural del clima organizacional dice que las interacciones de los individuos que comparten una cultura organizacional generan una atmósfera condicionada por dichos principios y reglas. Romero et al. (2015) en el trabajo realizado sobre medio organizacional tiene como objeto medir el desenvolvimiento de los servidores considerando el talento humano, realizado en la Universidad Técnica de Manabí-Ecuador, el estudio se realizó para reconocer los elementos que tienen primacía al interior de las instituciones como son el espacio laboral, las peculiaridades desarrollar el liderazgo, responsables de asumir decisiones de decisiones, formas colaborativas de trabajo y ética profesional como elementos constituyentes del clima laboral, factores educativos y 
culturales que hacen posible o imposible el proceso de valoración del potencial del talento humano.Gestión.Org, (2020) Las organizaciones se encuentran en búsqueda perenne de mejorar su productividad y alcance en la prestación de servicios, donde el clima institucional tiene una función importante, comprende las acciones que se dan en el medio laboral y determina la unión o el obstáculo dentro de la organización.

Los resultados de las dimensiones de la variable clima organizacional nos asigna los siguientes datos dimensión motivación en el trabajo arrojó un valor medio de 66,7\% considerado como muy bueno, este resultado nos indica la relación significativa con la variable gestión de talento y conflictos resultados que son apoyados con los estudios de (Toulson \& Smith, 1994) "Existe evidencia que sugiere que el clima organizacional puede influir tanto en el desempeño laboral como en la satisfacción de los empleados" si hay colaboradores contentos, se pueden promover como logros de atmósferas adecuadas al interior de la organización. (Madero Gómez \& Olivas Luján, 2016) tuvo la intensión de explorar los aspectos del comportamiento organizacional en personas que se estrenan en su vida laboral refiriéndose a la motivación menciona a Arrieta y Navarro (2008) como la fortaleza psicológica que actúa desde el interior de cada ser humano que indican la dirección del comportamiento, "de los niveles de esfuerzo y de persistencia cuando la conducta no está sujeta a fuertes presiones y limitaciones situacionales" poniendo énfasis en la interacción entre el ser (intereses, necesidades y valores) y el medio (tipo de tareas, condiciones laborales). Bajo estas condiciones y por los estudios ya sustentado concluimos que colaboradores motivados en la organización generan climas saludables para la organización.

El resultado de la dimensión liderazgo de la variable clima organizacional arroja un valor alto de 79,2\% considerado como muy bueno este resultado nos indica que para los docentes de las escuelas públicas la dimensión liderazgo tiene una relación significativa con la gestión del talento y conflictos, el resultado refiere la interacción realizada con diferentes grupos humanos al interior de las organizaciones educativas donde un papel relevante corresponde al liderazgo que en opinión de (Sills, 1968) ser líder consiste en hacer una dirección compartida, además implica que el grupo cuya interrelación es influenciada por el liderazgo tienen un objetivo común o al menos objetivos compartidos así mismo, Hollander y Julian (1964), señalan que los líderes guían positivamente hacia el logro de metas trazadas por la organización. En este sentido en las organizaciones 
educativas se miden el liderazgo directivo y el liderazgo pedagógico ambos gestionando el manejo de grupos humanos que se miden en un sólo resultado organizacional.

El resultado la dimensión comunicación de la variable clima organizacional nos arroja un valor alto de $73,3 \%$ considerado como muy bueno, esto nos indica que para los docentes de las escuelas públicas existe una relación significativa entre la dimensión comunicación y las variables gestión de talento y conflictos, este resultado la apoyamos en los resultados de Según (Chiavenato, 2007) una corporación es competitiva cuando los colaboradores se encuentran laborando en el mejor lugar, con las mejores circunstancias de salubridad, higiene y condiciones de seguridad psicológica y social con un orden fabuloso, en efecto motivados y con la más alta productividad. La gestión de la sumatoria de la calidad organizacional es consecuencia de la optimización de la excelencia humana y está relacionado de lo bien que se siente un colaborador en la organización. (Padhi, 2012) mencionado por (Herbert Ferrara, 2013) los directores o líderes se deben caracterizarse por estar abiertos a recibir información, a escuchar con eficiencia sugerencias y o alcances de los colaboradores dentro de la organización de manera que éstos se sientan cómodos proporcionando comentarios y críticas constructivas generando un nivel de seguridad en su comunicación.

\section{Análisis inferencial de las variables: Hipótesis general}

Ho: La gestión de talento y conflictos no tienen influencia significativamente en el clima organizacional de las escuelas públicas.

Ha: La gestión de talento y conflictos tienen influencia significativamente en el clima organizacional de las escuelas públicas.

La contrastación de la hipótesis se realizó por el algoritmo de regresión logística multinomial para dos variables independientes y una dependiente. A un nivel de significancia del 5\% (nivel de confianza es del 95\%).

Tabla 7 Información de la bondad de ajuste del modelo

\begin{tabular}{lccc}
\hline & Chi cuadrado & Gl & Significancia \\
\hline Pearson & 0.920 & 3 & $\mathbf{0 . 8 2 1}$ \\
Desviación & 1.495 & 3 & $\mathbf{0 . 6 8 4}$ \\
\hline
\end{tabular}

Fuente: Elaboración propia, 2021. 
Se observó en la prueba de bondad de ajuste que las significancias evidencian un valor mayor a 0.05 , en consecuencia, se confirma que los datos se ajustan correctamente al modelo (regresión logística multinomial).

Tabla 8 Información de ajuste del modelo

\begin{tabular}{cccc} 
Modelo & Chi cuadrado & Gl & Significancia \\
\hline Final & 97.172 & 2 & $\mathbf{0 . 0 0 0}$ \\
\hline
\end{tabular}

Fuente: Elaboración propia, 2021.

En resumen, el modelo de regresión logística multinomial para evaluar la influencia de las variables independientes sobre la variable dependiente, la cual resultó estadísticamente significativo $\mathrm{p}<0.050(\mathrm{p}=0.000)$. Significando el rechazo de la hipótesis nula y aceptando la hipótesis planteada por el investigador (hipótesis alternativa). Por lo tanto, se puede concluir que las variables gestión de talento y conflictos tienen influencia significativa en el clima organizacional.

Tabla 9 Información del pseudo R cuadrado del modelo

\begin{tabular}{lccc}
\hline & Cox y Snell & Nagelkeke & Mc Fadden \\
\hline Pseudo R cuadrado & 0.33 & $\mathbf{0 . 5 5 1}$ & 0.437
\end{tabular}

Fuente: Elaboración propia, 2021

Asimismo, se midió la calidad del ajuste utilizando el pseudo R cuadrado de Nagelkerke, con lo que se concluye que la variable dependiente es explicada en un $55.1 \%$ por las variables independientes, considerando esta influencia como aceptable (moderada).

\section{Hipótesis especifica $\mathrm{N}^{\circ} 1$}

Ho: La gestión de talento y conflictos no tienen influencia significativamente en la motivación en el trabajo de los docentes de las escuelas públicas.

Ha: La gestión de talento y conflictos tienen influencia significativamente en la motivación en el trabajo de los docentes de las escuelas públicas.

La contrastación de la hipótesis se realizó por el algoritmo de regresión logística multinomial A un nivel de significancia del 5\% (nivel de confianza es del 95\%).

Tabla 11 Información de la bondad de ajuste del modelo

\begin{tabular}{lccc}
\hline & Chi cuadrado & Gl & Significancia \\
\hline Pearson & 3.470 & 6 & $\mathbf{0 . 7 4 8}$ \\
Desviación & 4.540 & 6 & $\mathbf{0 . 6 0 4}$
\end{tabular}

Fuente: Elaboración propia, 2021. 
Se observó en la prueba de bondad de ajuste que las significancias evidencian un valor mayor a 0.05 , en consecuencia, se confirma que los datos se ajustan correctamente al modelo.

Tabla 12 Información de ajuste del modelo

\begin{tabular}{cccc}
\hline Modelo & Chi cuadrado & Gl & Significancia \\
\hline Final & 41.050 & 4 & $\mathbf{0 . 0 0 0}$
\end{tabular}

Fuente: Elaboración propia, 2021

En resumen, se utilizó el modelo de regresión logística multinomial para evaluar la influencia de las variables independientes sobre la dimensión de la variable dependiente (motivación en el trabajo), la cual resultó estadísticamente significativo $\mathrm{p}<0.050$ $(\mathrm{p}=0.000)$. Significando el rechazo de la hipótesis nula y aceptando la hipótesis planteada por el investigador (hipótesis alternativa). Por lo tanto, se puede concluir que las variables gestión de talento y conflictos tienen influencia significativa en la dimensión motivación en el trabajo del clima organizacional de las escuelas públicas.

Tabla 13 Información del pseudo R cuadrado del modelo

\begin{tabular}{lccc}
\hline & Cox y Snell & Nagelkeke & Mc Fadden \\
\hline Pseudo R cuadrado & 0.157 & $\mathbf{0 . 2 0 3}$ & 0.115 \\
\hline
\end{tabular}

Fuente: Elaboración propia, 2021

Asimismo, se midió la calidad del ajuste utilizando el Pseudo R cuadrado de Nagelkerke, con lo que se concluye que la variable dependiente (motivación en el trabajo) es explicada en un $20.3 \%$ por las variables independientes (gestión de talento y conflictos), considerando esta influencia como baja, pero aceptable.

\section{Hipótesis específica $\mathbf{N}^{\circ} 2$}

Ho: La gestión de talento y conflictos no tienen influencia significativamente en el liderazgo de las escuelas públicas.

Ha: La gestión de talento y conflictos tienen influencia significativamente en el liderazgo de las de las escuelas públicas.

La contrastación de la hipótesis se realizó por el algoritmo de regresión logística multinomial para dos variables independientes y una dimensión de la variable dependiente (liderazgo). A un nivel de significancia del 5\% (nivel de confianza es del $95 \%)$.

Tabla 15 Información de la bondad de ajuste del modelo 


\begin{tabular}{lccc}
\hline & Chi cuadrado & Gl & Significancia \\
\hline Pearson & 0.783 & 3 & $\mathbf{0 . 8 5 4}$ \\
Desviación & 0.806 & 3 & $\mathbf{0 . 8 4 8}$ \\
\hline
\end{tabular}

Fuente: Elaboración propia, 2021

Se observó en la prueba de bondad de ajuste que las significancias evidencian un valor mayor a 0.05 , en consecuencia, se confirma que los datos se ajustan correctamente al modelo.

Tabla 16 Información de ajuste del modelo

\begin{tabular}{cccc}
\hline Modelo & Chi cuadrado & Gl & Significancia \\
\hline Final & 87.537 & 2 & $\mathbf{0 . 0 0 0}$ \\
\hline
\end{tabular}

Fuente: Elaboración propia, 2021

En resumen, se utilizó el modelo de regresión logística multinomial para evaluar la influencia de las variables independientes (gestión de talento y conflictos) sobre la dimensión de la variable dependiente (liderazgo), la cual resultó estadísticamente significativo $\mathrm{p}<0.050(\mathrm{p}=0.000)$. Significando el rechazo de la hipótesis nula y aceptando la hipótesis planteada por el investigador (Hipótesis Alternativa).

Por lo tanto, se puede concluir que las variables gestión de talento y conflictos tienen influencia significativa en la dimensión liderazgo de la variable dependiente en las escuelas públicas.

Tabla 17 Información del pseudo R cuadrado del modelo

\begin{tabular}{lccc}
\hline & Cox y Snell & Nagelkeke & Mc Fadden \\
\hline Pseudo R cuadrado & 0.306 & $\mathbf{0 . 4 7 7}$ & 0.356 \\
\hline
\end{tabular}

Fuente: Elaboración propia, 2021

Asimismo, se midió la calidad del ajuste utilizando el Pseudo R cuadrado de Nagelkerke, con lo que se concluye que la dimensión liderazgo de la variable dependiente es explicada en un $47.7 \%$ por las variables independientes, considerando esta influencia como aceptable (moderada).

\section{Hipótesis específica $\mathrm{N}^{\circ}$ :}

Ho: La gestión de talento y conflictos no tienen influencia significativa en la comunicación de las escuelas públicas.

Ha: La gestión de talento y conflictos tienen influencia significativa en la comunicación de las escuelas públicas. 
La contrastación de la hipótesis se realizó por el algoritmo de regresión logística multinomial para dos variables independientes y una dimensión de la variable dependiente (comunicación). A un nivel de significancia del 5\% (nivel de confianza es del 95\%).

Tabla 19 Información de la bondad de ajuste del modelo

\begin{tabular}{lccc}
\hline & Chi cuadrado & Gl & Significancia \\
\hline Pearson & 0.202 & 3 & $\mathbf{0 . 9 7 7}$ \\
Desviación & 0.288 & 3 & $\mathbf{0 . 9 6 2}$
\end{tabular}

Fuente: Elaboración propia, 2021

Se observó en la prueba de bondad de ajuste que las significancias evidencian un valor mayor a 0.05 , en consecuencia, se confirma que los datos se ajustan correctamente al modelo (regresión logística multinomial).

Tabla 20 Información de ajuste del modelo

\begin{tabular}{cccc}
\hline Modelo & Chi cuadrado & Gl & Significancia \\
\hline Final & 87.896 & 2 & $\mathbf{0 . 0 0 0}$ \\
\hline
\end{tabular}

Fuente: Elaboración propia, 2021

En resumen, se utilizó el modelo de regresión logística multinomial para evaluar la influencia de las variables independientes sobre la dimensión comunicación de la variable dependiente, la cual resultó estadísticamente significativo $\mathrm{p}<0.050 \quad(\mathrm{p}=0.000)$. Significando el rechazo de la hipótesis nula y aceptando la hipótesis planteada por el investigador (hipótesis alternativa). Por lo tanto, se puede concluir que las variables gestión de talento y conflictos tienen influencia significativa de las escuelas públicas.

Tabla 21 Información del Pseudo R cuadrado del modelo

\begin{tabular}{lccc}
\hline & Cox y Snell & Nagelkeke & Mc Fadden \\
\hline Pseudo R cuadrado & 0.307 & $\mathbf{0 . 4 4 7}$ & 0.316 \\
\hline
\end{tabular}

Fuente: Elaboración propia, 2021

Asimismo, se midió la calidad del ajuste utilizando el Pseudo R cuadrado de Nagelkerke, con lo que se concluye que la dimensión comunicación de la variable dependiente es explicada en un $44.7 \%$ por las variables independientes (gestión de talento y conflictos), considerando esta influencia como aceptable (moderada).

Se ha demostrado la correlación significativa entre la gestión de talento y conflictos en el clima organizacional de las escuelas públicas como lo demuestra la prueba del pseudo $\mathrm{R}$ 
cuadrado, la misma que expresa la dependencia porcentual de la gestión del talento y manejo de conflictos en el clima organizacional, para lo cual se tiene al coeficiente de Nagelkerke, implicando que la variabilidad del clima organizacional depende el 55,1\% de la gestión del talento y conflictos. En relación al primer objetivo específico, los resultados indican que la variabilidad de la gestión del talento y manejo de conflictos influyen en el clima organizacional, como se verifica con la prueba del pseudo $\mathrm{R}$ cuadrado, en la cual se expresa la dependencia porcentual de la Gestión del talento y manejo de conflictos en la motivación en el trabajo de las escuelas públicas, para lo cual se tiene al coeficiente de Nagelkerke, implicando que la variabilidad de la motivación en el trabajo depende en un $20.3 \%$ de la gestión del talento humano y el manejo de conflictos. Respecto al segundo objetivo específico, los resultados indican que la gestión del talento y conflictos influyen en el liderazgo, como se verifica con la prueba del pseudo $\mathrm{R}$ cuadrado, en la cual se expresa la dependencia porcentual de la Gestión del talento y conflictos en el liderazgo de las escuelas publicas, para lo cual se tiene al coeficiente de Nagelkerke, implicando que la variabilidad del liderazgo depende el 47,7\% de la gestión del talento y conflictos. En referencia al tercer objetivo específico, los resultados indican que la gestión del talento y conflictos influyen en la comunicación, como se verifica con la prueba del pseudo R cuadrado, en la cual se expresa la dependencia porcentual de la gestión del talento y conflictos en la comunicación de las escuelas públicas, para lo cual se tiene al coeficiente de Nagelkerke, implicando que la variabilidad de la comunicación depende $44,7 \%$ de la gestión del talento y conflictos.

\section{CONCLUSIÓN O CONSIDERACIONES FINALES}

- Se determinó que la gestión de talento humano y conflictos inciden significativamente en el clima organizacional, en un $87.9 \%$.

- Se determinó que la gestión de talento humano y conflictos inciden significativamente en la motivación en el trabajo, en un 66,7\%.

- Se determinó que la gestión de talento humano y conflictos inciden significativamente en el liderazgo, en un 79,2\%.

- Se determinó que la gestión de talento humano y conflictos inciden significativamente en la comunicación, en un $79,2 \%$.

\section{REFERENCIAS BIBLIOGRAFICAS}

.Org, G. (2020). Obtenido de https://www.gestion.org/que-es-el-clima-organizacional/ 
Acosta, B., \& Venegas, C. (2010). Clima organizacional en una empresa cervecera: un estudio exploratorio. Gale Onefile Psicología.

Bowie, N. (2013). Integridad organizacional y climas morales. El manual de la ética empresarial de Oxford, 701-724.

Bustamante Ubilla, M. A., Grandón Avendaño, M. L., \& Lapo Maza, M. d. (2015). Caracterización del clima organizacional en hospitales de alta complejidad en Chile. ELSEVIER /estudios generales.

Cardona Aarbelaez, D., Lamadrid Villareal , J., \& Brito Carrillo, C. (2018). La gestión y dirección del talento humano desde el análisis sobre clima organizacional y sus dimensiones. Un estudio de caso. Dialnet. doi:DOI 10.22519/22157360.1185

Chiavenato, I. (2007). Administración y recursos humanos. Mexico.

Chiavenato, I. (2009). Gestión del Talento Humano. Mexico.

Eckart, R., Lepak, D. P., \& Boselie, P. (2017). Integrating strategic human capital and strategic human resource management. The International Journal of Human Resource Management.

Herbert Ferrara, M. (2013). Gestión del ciclo de vida del producto. Gestión del ciclo de vida del producto. Manual de gestión de la innovación de Gale Business Insights.

Jee Kim, E., \& Sunyoung, P. (2018). Changers' Organizational Commitment and Job Satisfaction. Journal of Career Development.

Madero Gómez, S. M., \& Olivas Luján, M. R. (2016). Análisis de los factores del comportamiento organizacional en jóvenes que están iniciando su carrera laboral. Redalyc.org. doi:https://doi.org/10.1016/j.estger.2015.12.001

Maxwell, G. (2014). Satisfacción Laboral. Libros Electrónicos de Gale.

Mc Donnell, A. (2011). Are you still fighting the "war for talent"? Reduce the gap between science and practice. Journal Of Business And Psychology.

Moran Jarqui, M. (2020). Estrés laboral como generador de conflictos escolares en el aula a nivel preescolar. Copyright of revista mexicana de orientación educativa is the property of centro de investigación.

Morrill, C. (2000). Uso de conflictos en organizaciones. Gale Onefile Psicología.

Piva Biagini, A., \& Padua da Silva, G. (2015). Nivel de calidad de vida laboral de los empleados de una clínica geriátrica. Gale Onefile. 
Romero Vélez, M., Verduga Pino, A., \& Macías Zambrano, N. (2015). Clima organizacional enfocado el desempeño del talento humano. Dialnet.

Sarabia De Ortega, L. (2014). Clima organizacional y procesos de reingenieria en una empresa de consumo masivo/organizational. Gale Onefile Informe Académico.

Sardón Ari, D. L., \& Sardón Ari, Z. Y. (2018). Estilos de manejo de conflictos y desempeño docente en instituciones educativas de nivel primario. Latindex.

Serra Fernández, G. (2021). Blog del Instituto de alto rendimiento. Obtenido de https://institutoaltorendimiento.com/gestiondeltalento/inicio/author/gonzalo/

Toulson, P., \& Smith, M. (1994). La relación entre el clima organizacional y las percepciones de los empleados sobre las prácticas de gestión de personal. Gale Onefile.

Yazdi , S., \& Yakhdani, M. F. (2017). La relación entre el clima organizacional, el compromiso laboral y el papel mediador de la conciencia laboral. Gale Onefile Referencias del Educador Completa. 